\title{
Malakoplakia of the Urinary Tract: A Benign Disease with a Possible Malignant Outeome
}

\section{JCR}

Wong SHL', Yeung VHW', Lee WK ${ }^{2}$, Lee YK' ${ }^{1}$, Chan MTY' ${ }^{1}$, Cheng CH' ${ }^{1}$ Chu PSK' ${ }^{1}$, Man CW' 'Division of Urology, Department of Surgery, Tuen Mun Hospital, Hong Kong; ${ }^{2}$ Department of Pathology, Tuen Mun Hospital, Hong Kong.

Abstract:

Introduction: Malakoplakia is a rare chronic inflammatory disease which commonly affects the urogenital tract. The exact pathogenesis is not fully understood, but some associated conditions which include immunodeficiency, malignancy or chronic bacterial infection, have been suggested. The definite diagnosis relies on identifying the Michaelis-Gutmann bodies microscopically. Case Report: A 62-year-old woman with poorly controlled diabetes mellitus presented with gross hematuria for a few months. Subsequent investigations showed a positive urine culture (Escherichia coli - E. coli), and flexible cystoscopy showed a $1 \mathrm{~cm}$ plaque-like lesion at the left posterior wall of the urinary bladder as well as several smaller, similar lesions noted at various different sites. Biopsy of the lesion showed a proliferation of macrophages $(C D 68+)$ with abundant, glandular cytoplasm and the presence of Michaelis-Gutmann bodies (PAS+, Von Kossa +), which was consistent with malakoplakia. Computed Tomography (CT) urogram showed no hydronephrosis, with good contrast excretion from both kidneys as well as satisfactory drainage down to the bladder. She was then treated with antibiotics, but still had on and off urinary tract infection. About a year later, she presented with urosepsis and acute renal failure, and was admitted to the urological ward. Urgent CT urogram showed bilateral hydronephrosis with both lower ureters distended with hyperdense contents (malakoplakia plaques). Emergency bilateral percutaneous nephrostomy ( $P C N)$ was performed. Her condition was stabilized with antibiotics, and her renal function improved with PCN drainage. Several treatment options were offered to the patient, but in view of her significant pre-morbid condition, she opted for bilateral long-term PCN. Conclusion: Malakoplakia was thought to be a treatable and relatively benign disease, but in some cases, it might result in renal failure due to bilateral ureteric involvement. Early diagnosis together with adequate antibiotic treatment is important in patients with malakoplakia, and they should be closely followed up to prevent future development of renal insufficiency.

Key words: Acute Kidney Injury, Hematuria, Hydronephrosis, Malakoplakia, Nephrostomy, Urinary bladder.

Corresponding Author: Dr. Wong Hoi Lung Simon

Email: simonwonghoilung@hotmail.com

Received: February 18, 2016 | Accepted: May 4, 2016 | Published Online: June 15, 2016

This is an Open Access article distributed under the terms of the Creative Commons Attribution License (creativecommons.org/licenses/by/3.0)

Conflict of interest: None declared | Source of funding: Nil | DOI: http://dx.doi.org/10.17659/01.2016.0063 


\section{Introduction}

Malakoplakia is a rare chronic inflammatory disease which was first described in 1902 by Michaelis and Gutmann [1], and is more common in females [2]. It commonly affects the genitourinary system with bladder being the most frequently affected organ [3]. Malakoplakia can be asymptomatic or present as hematuria, bladder mass, nodule or soft yellow plaque. [4]. The exact pathogenesis is not fully understood, but most patients have urine infections by $E$. coli, and $40 \%$ of them are associated with some type of immunodeficiency or malignancy [2]. We hereby present an unusual case of a woman who was diagnosed to have bladder malakoplakia, which later progressed to renal failure within a period of three years.

\section{Case Report}

A 62-year-old woman with hypertension, hyperlipidemia, poorly controlled diabetes and a history of stroke, first presented to her primary care physician with on and off hematuria, urinary urgency and frequency in November 2012. Urine culture revealed $E$. coli, and she was treated with antibiotics. However, she had persistent dysuria and bacteriuria, and therefore she was referred to urologist for further management. For the hematuria workup, no acid fast bacilli were found in the early morning urine samples, and there was no evidence of malignancy in the urine cytology specimens. CT urogram with contrast showed that both kidneys were normal in size and contours with no hydronephrosis or hydroureter. No abnormal calcification was seen in both kidneys, pelvicalyceal systems, ureters and urinary bladder. The renal parenchyma showed normal attenuation and enhancement pattern, with normal excretion of both kidneys. However, an $8.3 \mathrm{x}$ $9.2 \mathrm{~mm}$ polypoid lesion was seen over left posterior wall of urinary bladder.

Cystoscopy and transurethral resection of bladder tumour (TURBT) was performed in March
2013. Intra-operatively, multiple patches of plaque-like lesions were found inside the bladder, and bilateral ureteric openings were intact. The most prominent one $1-\mathrm{cm}$ plaque-like lesion was located at the left lateral wall of bladder, and all lesions were completely resected and sent for section. Microscopically, the resected bladder lesions showed a proliferation of macrophages $(C D 68+)$ with abundant, glandular cytoplasm, and the presence of Michealis-Gutmann bodies (PAS+, Von Kossa+), which was consistent with malaoplakia. There was no evidence of malignancy [Fig.1-2]. Her hematuria subsided after the operation, and was discharged uneventfully.

The patient was admitted again in January 2015 with dysuria, and subsequently developed septic shock. Urethral catheterisation yielded turbid urine. Serum creatinine was raised to $298 \mu \mathrm{mol} / \mathrm{L}$ when compared to her baseline level of $150 \mu \mathrm{mol} / \mathrm{L}$. Bilateral ultrasonic evidence of hydronephrosis was detected which was more severe on the left side. Urgent CT urogram showed no urolithiasis, but the urothelium of the bilateral pelvocalyceal system and ureter was thickened together with strandings around the ureters, which was compatible with an obstructive and infective process [Fig.3].

Emergency urinary diversion for urosepsis due to obstructive uropathy was attempted by

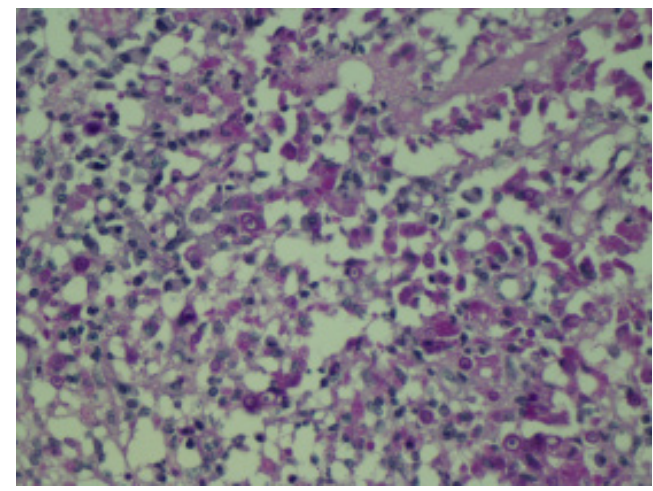

Fig. 1: PAS stain: Michealis-Guttmann bodies (magenta stained, ring-like with a central dot). 
cystoscopic double J stent insertion. However, urinary bladder was markedly inflamed with edematous mucosa, and the right ureteric orifice could not be identified. The left ureteric orifice was successfully cannulated, but the retrograde pyelogram showed a hairline stricture at distal ureter, which the contrast and guidewire could not negotiate through. Thus, bilateral PCNs was performed, and the sepsis was controlled after PCN insertion and antibiotics.

She was examined again with cystoscopy and flexible ureterorenoscopy in April 2015. Cystoscopy showed a chronically infected bladder, and both ureteric orifices were not seen. Right antegrade ureterorenoscopy showed multiple polypoid lesions at renal pelvis and downward to upper ureter, and the ureteroscope could not pass beyond the site of obstruction. Bilateral antegrade pyelogram via percutaneous nephrostomy was performed afterward showed left ureteric complete obstruction at L4 level and right ureteric multiple filling defect from L4 to vesicoureteric junction [Fig.4].

Video urodynamic study was arranged subsequently, and it showed a very small functional bladder capacity $(\sim 20 \mathrm{ml})$, and urine leakage was noted if more than $20 \mathrm{ml}$ of contrast instilled. This reflected that the malakoplakia involved both the urinary bladder and bilateral ureters causing renal impairment and sepsis. Various treatment options including major bladder and ureteric reconstruction by augmentation, enterocystoplasty and ileal interposition were offered to the patient, but after thorough discussion with nephrologist, because her calculated GFR was $20 \mathrm{ml} / \mathrm{min}$, she opted for long term bilateral PCNs in view of her significant comorbidities.

\section{Discussion}

Malakoplakia is a rare disease, and the total number of affected patients in the United States is less than 500. The disease is female predominance

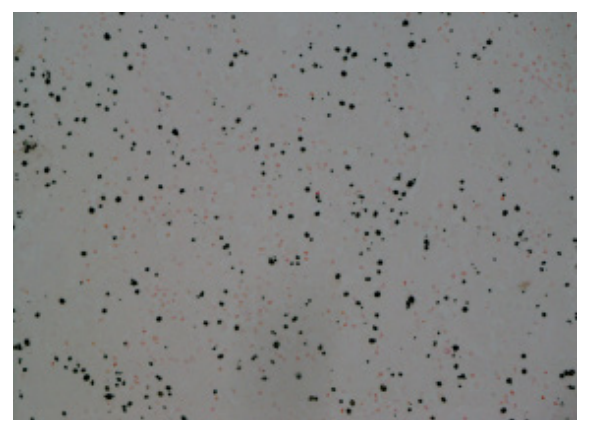

Fig.2: Von-Kossa stain for calcium - MichealisGuttmann bodies (black dot).

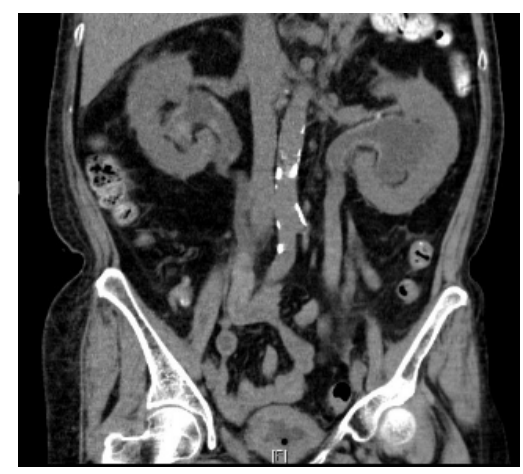

Fig.3: Urgent CT urogram showed no urolithiasis, but the urothelium of the bilateral pelvocalyceal system and ureter was thickened together with strandings around the ureters.

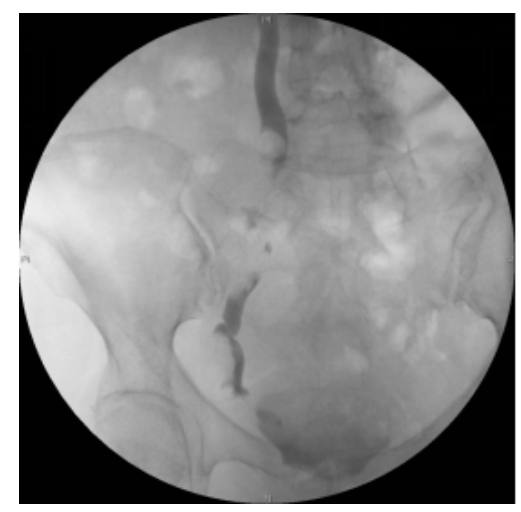

Fig.4: Antegrade pyelogram via percutaneous nephrostomy performed showed right ureteric multiple filling defects (ureteric strictures) from L4 to vesicoureteric junction. 
(female-male ratio of $4: 1$ ), but there is no particular racial predilection $[5,6]$. Malakoplakia tends to occur in an older age group (average: 50 years old), with a range from 6 weeks to 85 years old [6].

The etiology of malakoplakia is not well understood, and several possible mechanisms have been suggested [6-9]. First, microorganisms play a role in the pathogenesis, in particularly E. coli, Proteus and Mycobacterium tuberculosis [6]. Secondly, an abnormal or altered immune response has been implicated in the pathogenesis, and an immunocompromised status is associated with the pathogenesis [7]. The third hypothesis is an abnormal macrophage response because of defective lysosomal function [8,9]. It is suggested that macrophages in malakoplakia are capable of phagocytosis but unable to digest the bacteria. Partially digested bacteria accumulate in macrophages and lead to deposition of calcium and iron on residual bacterial glycolipid. The resulting basophilic structure, the Michaelis-Gutmann body, is considered pathogomonic for malakoplakia $[6,7,10]$.

The urinary tract is the most commonly involved site of malakoplakia. Wielenberg et al. [11] in a review of 153 cases, found 89 cases (58\%) that involved the urinary tract, out of which $63(40 \%)$ occurred in the urinary bladder. Next to the bladder, commonly involved urinary sites are the kidneys, ureters, and renal pelvis. Although malakoplakia of urinary tract is common, its presentation can be variable. It can be asymptomatic, or presents as painless hematuria, urinary tract infection, septicaemia, hydronephrosis, acute renal failure or complications of end stage renal disease.

Bacterial infection and immunocompromised status are believed to be related to the development of malakoplakia. Antibiotic against E. coli is an effective treatment of malakoplakia
[12]. The prolonged course of antibiotics or combined use of quinolone antibiotics together with trimethoprim-sulfamethoxazole has been shown to improve the outcome of malakoplakia $[5,7,12,13]$. As the etiology of malakoplakia was related to immunosuppression, several studies report that the disease regressed when the patient was rendered into a less immunosuppressed state $[7,9,12]$.

The outcome of malakoplakia is variable. Some reported cases had resolved condition after antibiotics and no further recurrence. There are several reported cases showed disease recurrence or progression causing life threatening complications. Table 1 shows the recent published reports on malakoplakia of the urinary tract.

This presentation aims at highlighting the possible malignant outcome of malakoplakia despite its previously known benign nature. Regular follow up is necessary to detect any possible recurrence of malakoplakia. Urine culture should be performed to confirm the complete eradications of bacterial infection. Prolonged course of antibiotics with the combination of quinolone and trimethoprimsulfamethoxazole may be required. Surveillance cystoscopy is recommended as it is able to exclude local recurrence or disease progression. Upper urinary tract imaging such as ultrasound of kidney or CT urogram is useful for disease surveillances.

\section{Conclusion}

Malakoplakia was thought to be a treatable and relatively benign disease, but in some cases, it might result in renal failure due to bilateral ureteric involvement. Early diagnosis together with adequate antibiotic treatment is important in patients with malakoplakia, and they should be closely followed up to prevent future development of renal insufficiency. 
Table 1: Recent Published Reports on malakoplakia of the Urinary Tract*

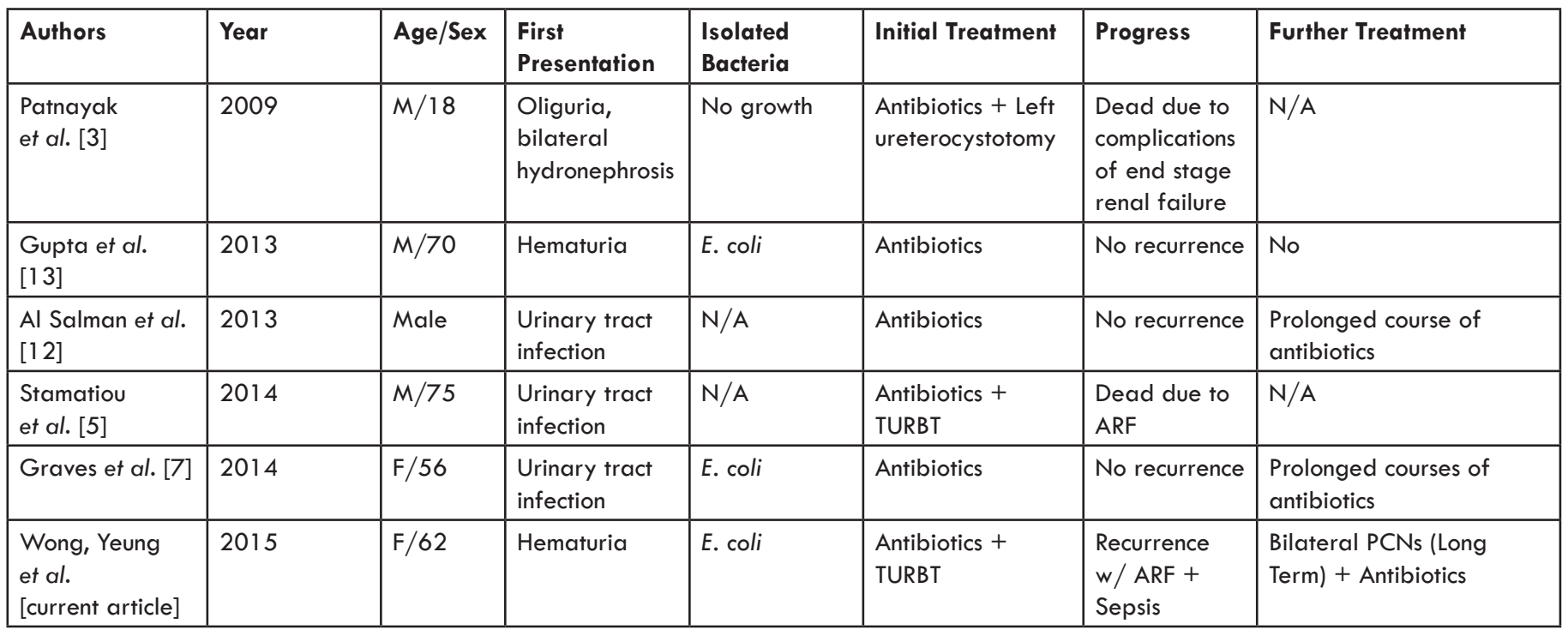

*TURBT $=$ Transurethral resection of bladder tumor, $\mathrm{ARF}=$ Acute renal failure, N/A $=$ Not Applicable

\section{References}

1. Michaelis L, Gutmann C. Einschlusse in blastentumoren. Z Klin Med. 1902;47:208-215.

2. Stanton MJ, Maxted W. Malacoplakia: a study of the literature and current concepts of pathogenesis, diagnosis and treatment. J Urol. $1981 ; 125: 139-146$.

3. Patnayak R, Reddy $M$, Subramanian S, Jena A, Ravisankar G, Dandu R. An unusual case of bilateral hydroureteronephrosis caused by uretero-vesico malakoplakia in a young male: a case report and review of the literature. Cases J. $2009 ; 2(1): 7527$.

4. Lee SL, Teo JK, Lim SK, Salkade HP, Mancer K. Coexistence of Malakoplakia and Papillary Urothelial Carcinoma of the Urinary Bladder. Int J Surg Pathol. 2015;23(7):575-578.

5. Stamatiou K, Chelioti E, Tsavari A, Koulia K, Papalexandrou A, Efthymiou E, et al. Renal Failure Caused by Malakoplakia lesions of the Urinary Bladder. Nephrourol Mon. 2014;6(4):e18522.

6. Yousef GM. Malakoplakia Outside The Urinary Tract. Arch Pathol Lab Med. 2007;131(2):297300.
7. Graves AL, Texler M, Manning L, Kulkarni H. Successful Treatment Of Renal Allograft And And Prolonged Antibiotic Therapy. Nephrology. 2014;19:18-21.

8. Rickert $\mathrm{CH}$, August $\mathrm{C}$, Brandt $\mathrm{M}$, Wagner $\mathrm{V}$, Paulus W. Cerebral Bladder Malakoplakia with Minimization of Immunosuppression malakoplakia associated with Escherichia coli infection. Acta Neuropathol (Berl). 2000;99:595598.

9. Govender D, Essa AS. Malakoplakia and tuberculosis. Pathology. 1999;31:280-283.

10. Lou TY, Teplitz C. Malakoplakia: pathogenesis and ultrastructural morphogenesis: a problem of altered macrophage (phagolysosomal) response. Hum Pathol. 1974;5:191-207.

11. Wielenberg AJ, Demos TC, Rangachari B, Turk T. Malacoplakia presenting as a solitary renal mass. Am J Roentgenol. 2004;1 83:1703-1705.

12. Al Salman, JM., Husain A. Renal Malakolpkia Followed By Chronic Myelogenous Leukaemia: Treatment Challenges Case Report And Literature Review. BMJ Case Rep. 2013 Jan 23;2013.

13. Gupta R, Mahajan A, Atri S, Gupta CL. Recurrent Painless Hematuria Secondary To Malacoplakia Of The Urinary Bladder. Urol J. 2013;10(1):82 1 823. 